\title{
Differential effects of emphysema on skeletal muscle fibre atrophy in hamsters
}

\author{
J.P. Mattson*, M.D. Delp\#, D.C. Poole
}

Differential effects of emphysema on skeletal muscle fibre atrophy in hamsters. J.P. Mattson, M.D. Delp, D.C. Poole. C) ERS Journals Ltd 2004.

ABSTRACT: Patients afflicted with emphysema demonstrate altered peripheral skeletal muscle fibre composition and atrophy. It is unknown whether these alterations are general to all skeletal muscles independent of function, phenotype or oxidative capacity. Therefore, the purpose of this investigation was to determine whether emphysema induces alterations in muscle fibre composition or atrophy in respiratory and locomotory muscles with diverse fibre types and metabolic profiles.

Fibre composition and cross-sectional area were measured in selected hindlimb muscles and diaphragm of hamsters following saline (control, $n=7$ ) or elastase (emphysema, $n=15)$ instillation.

Excised lung volume increased $145 \%$ with emphysema. Fibre composition was largely unaltered, with the exception of a $13 \%$ reduction in IIB fibres in the tibialis anterior muscle of emphysema animals. Type I fibre size was also mainly unaltered, except for a diminished cross-sectional area in plantaris muscle. However, fibre cross-sectional area of fast-twitch types IIA, IIX and/or IIB fibres was reduced in the caudal biceps femoris, vastus lateralis, tibialis anterior, gastrocnemius and plantaris muscles of emphysema animals. In contrast, there was a trend for emphysema to increase the cross-sectional area of type IIA fibres in the diaphragm.

These data demonstrate that emphysema-induced atrophy primarily affects locomotory muscles, independent of phenotype or oxidative capacity. Eur Respir J 2004; 23: 703-707.

One of the primary consequences of emphysema (EMP) or chronic obstructive pulmonary disease (COPD) is a decrement in work capacity. Numerous peripheral mechanisms have been proposed to contribute to the dysfunction, including reductions in peripheral skeletal muscle blood flow, hypoxemia and alterations in metabolism. Previous investigations have demonstrated reductions in peripheral skeletal muscle oxidative capacity in COPD patients [1-4] and hamsters induced with EMP [5], which could alter metabolism by activating anaerobic pathways to generate the required energy production. Such a shift would predispose skeletal muscle to greater fatigability and impair exercise tolerance. In addition, COPD patients with reduced work capacity present with alterations in peripheral skeletal muscle composition and structure, including altered fibre composition [4, 6, 7] and myosin heavy chain (MyHC) expression [8, 9], atrophy of peripheral skeletal muscle type I and/or II fibres [7, 10, 11], and reductions in lean body mass [12]. Therefore, altered metabolic pathways, fibre transformation and fibre atrophy appear to underlie problems of enhanced skeletal muscle fatigability, reduced force production and exercise intolerance in patients with COPD.

What remains unresolved is whether pulmonary disorders, such as EMP, have a generalised effect on skeletal muscle (locomotory and respiratory), or whether fibre transformation and atrophy occur in skeletal muscles composed of a given fibre type or oxidative capacity. Knowledge of such could provide the basis for targeting specific therapeutic
*College of Health, University of Utah, Salt Lake City, UT, " Depts of Health and Kinesiology and Medical Physiology, and the Cardiovascular Research Institute, Texas A\&M University, College Station, TX, and Depts of Anatomy, Physiology and Kinesiology, Kansas State University, Manhattan, KS, USA.

Correspondence: J.P. Mattson, 1850 East 250 South, Room 241, College of Health, University of Utah, Salt Lake City, UT84112-0920 USA.

Fax: 18015853992

E-mail: jmattson@hsc.utah.edu

Keywords: Chronic obstructive pulmonary disease, muscle composition, muscle fibre cross-sectional area

Received: September 152003

Accepted after revision: January 82004

This study was supported by USA Public Health Service Grant HL-50306 from the National Institutes of Health and American Lung Grant RG-013-N. interventions aimed at reversing these alterations. Therefore, utilising an accepted animal model, the purpose of this investigation was to determine whether EMP alters muscle fibre composition or induces atrophy in respiratory and locomotory muscles with diverse fibre types and metabolic profiles. Based on previously reported alterations in muscle oxidative capacity [5], the authors hypothesised that EMP would induce muscle-specific reductions in the proportion of type I fibres and increases in type II fibres. Furthermore, based upon the work of HuGHES et al. [10] suggesting an increased susceptibility of type II fibres to atrophy, the authors hypothesised muscle-specific atrophy of type II fibres.

\section{Methods}

Protocols used in this investigation were approved by the University of Utah Institutional Animal Care and Use Committee. They conform to the Guide for the Care and Use of Laboratory Animals published by the USA National Institutes of Health.

\section{Emphysema model}

Adolescent male Syrian Golden hamsters (100-120 g) were housed in $7.5 \times 8.5$ inch cages, maintained on a 12-h light-dark cycle, and supplied rodent chow and water ad libitum. Following a 1-week habituation period, animals were randomly 
divided into control (CON) and EMP groups. Under deep ketamine/xylazine $\left(150 / 7.5 \mathrm{mg} \cdot \mathrm{kg}^{-1}\right.$ intramuscular) anaethesia, either saline $\left(0.3 \mathrm{~mL} \cdot 100 \mathrm{~g}\right.$ body weight $\left.^{-1}\right)$ or porcine elastase (25 IU $\cdot 100$ g body weight $^{-1}$ (Sigma Chemical, St. Louis, MO, USA) in $0.3 \mathrm{~mL}$ of normal saline) was instilled intratracheally as previously described [5].

\section{Tissue harvesting}

Six months following injection, hamsters were euthanised, and the lungs, caudal biceps femoris, vastus lateralis, gastrocnemius, soleus, plantaris, tibialis anterior and diaphragm (costal and crural portions) muscles were excised. After being weighed, a 5-mm midbelly portion was dissected from each muscle, frozen in melting isopentane and stored at $-70^{\circ} \mathrm{C}$. A saline displacement technique was used to measure excised lung volume at $0 \mathrm{cmH}_{2} \mathrm{O}$ airway pressure.

\section{Immunohistochemical analysis}

A cryostat microtome was used to cut serial transverse cross-sections $(8-10 \mu \mathrm{m})$ of muscle. Fibre type identification was performed as previously described [13]. Briefly, sections were cold fixed $(50 \mathrm{~mL}$ of $37 \%$ zinc formalin and $370 \mathrm{~mL} 95 \%$ ethanol and $25 \mathrm{~mL}$ glacial acetic acid) for $5 \mathrm{~min}$. Slides were hydrated for $10 \mathrm{~min}$ in phosphate-buffered saline (PBS), then blocked (InnoGenex, San Ramon, CA, USA (\#BS-1310-25)) for $5 \mathrm{~min}$ at room temperature. After blocker removal, antibodies (American Type Culture Collection, Nanassas, VA, USA) to MyHCs, type I (BA-D5), type IIA (SC-71), or type IIB (BF-F3), were added to muscle sections, and the slides were incubated at $4{ }^{\circ} \mathrm{C}$ overnight. Following incubation, slides underwent a $2 \times 10$-min wash in PBS, then a biotinylated goat antimouse immunoglobulin secondary antibody (InnoGenex \#AS-2400-16) was added to the sections for $20 \mathrm{~min}$ at room temperature. Slides were washed and incubated for 20 min in streptavidin alkaline phosphatase conjugate (InnoGenex \#CJ-1002-86) at room temperature. Conjugate was removed by washing and a solution of naphthol phosphate buffer (InnoGenex \#BS-080204) and Fast Red dye (InnoGenex \#CH-0802-06) was added to the sections and incubated for colour development. Sections were counterstained with Mayer's Haematoxylin and mounted with Glycergel (Dako, Carpinteria, CA, USA).

\section{Determination of muscle fibre composition and cross-sectional area}

Muscle cross-sections were divided into four to five nonoverlapping regions as previously described [13, 14]. Representative fascicles with fibres cut perpendicular to their long axes were measured with an image processing system (Bioquant Nova Prime, Nashville, TN, USA). A minimum of five fibres of each type were measured for cross-sectional area (CSA) in each divided region. Exceptions to this procedure were made when only a few fibres of a given fibre type were present in a muscle section. Under this circumstance, fibre CSA was measured in all the fibres present of that type. In most cases, 750-1,000 fibres were typed per muscle cross-section and CSA of $\sim 100$ fibres per type per muscle were determined.

\section{Statistical analysis}

Lung volume, fibre CSA and per cent fibre type were compared between groups using the unpaired t-test. Data are presented as mean $\pm \mathrm{SD}$ and significance was accepted at $\mathrm{p} \leqslant 0.05$.

\section{Results}

\section{Emphysema condition}

The final body weight of EMP ( $\mathrm{n}=15,130 \pm 12 \mathrm{~g})$ was lower $(\mathrm{p}=0.04)$ than $\mathrm{CON}(\mathrm{n}=7,142 \pm 11 \mathrm{~g})$ hamsters. The presence of lung pathology and air trapping was supported by the large increase $(145 \% ; \mathrm{p}<0.01)$ of lung volume in EMP $(2.8 \pm$ $0.5 \mathrm{~mL})$ versus $\mathrm{CON}(1.1 \pm 0.1 \mathrm{~mL})$ animals.

\section{Fibre composition and cross-sectional area}

In tibialis anterior muscle of EMP hamsters, there were $12 \%$ fewer type IIB fibres than in CON hamsters (table 1 ). In addition, there was a trend for reduced IIA fibre composition in gastrocnemius $(6 \% ; \mathrm{p}=0.07)$ and plantaris $(7 \% ; \mathrm{p}=0.08)$ muscles of EMP compared to CON hamsters. However, there were no other differences in fibre composition between EMP and CON hamsters nor was there any significant correlation between lung pathology and skeletal muscle phenotype.

CSA of type I fibres was reduced in the plantaris of EMP hamsters compared with CON (table 2). CSA of type IIA fibres was reduced in the caudal biceps femoris, vastus lateralis and plantaris. CSA of type IIX fibres was reduced in the caudal biceps femoris, vastus lateralis, gastrocnemius and plantaris. CSA of type IIB fibres was reduced in the caudal biceps femoris, vastus lateralis and tibialis anterior. There was also a trend for CSA of type IIX fibres to be reduced $(p=0.06)$ in the tibialis anterior muscle of hamsters with EMP compared to CON. EMP had no significant effect on soleus or diaphragm fibre CSA nor was there any significant

Table 1. - Per cent muscle fibre composition of type I, IIA, IIX, and IIB fibres

\begin{tabular}{|c|c|c|c|c|}
\hline Muscle & I & IIA & IIX & IIB \\
\hline \multicolumn{5}{|c|}{ Biceps femoris, caudal } \\
\hline $\mathrm{CON}$ & $3 \pm 4$ & $13 \pm 1$ & $18 \pm 7$ & $66 \pm 5$ \\
\hline EMP & $3 \pm 4$ & $9 \pm 7$ & $20 \pm 10$ & $68 \pm 10$ \\
\hline \multicolumn{5}{|c|}{ Vastus lateralis } \\
\hline $\mathrm{CON}$ & $3 \pm 4$ & $31 \pm 8$ & $57 \pm 14$ & $9 \pm 9$ \\
\hline EMP & $7 \pm 5$ & $35 \pm 10$ & $50 \pm 19$ & $8 \pm 10$ \\
\hline \multicolumn{5}{|c|}{ Gastrocnemius } \\
\hline $\mathrm{CON}$ & $10 \pm 5$ & $31 \pm 5$ & $59 \pm 8$ & $0 \pm 0$ \\
\hline EMP & $17 \pm 10$ & $25 \pm 6^{\#}$ & $55 \pm 14$ & $3 \pm 5$ \\
\hline \multicolumn{5}{|l|}{ Soleus } \\
\hline $\mathrm{CON}$ & $80 \pm 7$ & $20 \pm 7$ & $0 \pm 0$ & $0 \pm 0$ \\
\hline EMP & $81 \pm 6$ & $19 \pm 6$ & $0 \pm 0$ & $0 \pm 0$ \\
\hline \multicolumn{5}{|l|}{ Plantaris } \\
\hline $\mathrm{CON}$ & $8 \pm 3$ & $69 \pm 4$ & $23 \pm 6$ & $0 \pm 0$ \\
\hline EMP & $12 \pm 4$ & $62 \pm 8^{\#}$ & $26 \pm 10$ & $0 \pm 0$ \\
\hline \multicolumn{5}{|c|}{ Tibialis anterior } \\
\hline $\mathrm{CON}$ & $7 \pm 4$ & $34 \pm 2$ & $41 \pm 8$ & $18 \pm 3$ \\
\hline EMP & $6 \pm 1$ & $37 \pm 5$ & $51 \pm 2$ & $6 \pm 3^{\circ}$ \\
\hline \multicolumn{5}{|c|}{ Diaphragm, costal } \\
\hline $\mathrm{CON}$ & $24 \pm 3$ & $47 \pm 7$ & $29 \pm 6$ & $0 \pm 0$ \\
\hline EMP & $24 \pm 7$ & $48 \pm 12$ & $28 \pm 11$ & $0 \pm 0$ \\
\hline \multicolumn{5}{|c|}{ Diaphragm, crural } \\
\hline $\mathrm{CON}$ & $17 \pm 8$ & $45 \pm 12$ & $38 \pm 14$ & $0 \pm 0$ \\
\hline EMP & $15 \pm 10$ & $49 \pm 11$ & $33 \pm 11$ & $3 \pm 6$ \\
\hline
\end{tabular}

Data are presented as mean \pm SD. CON: control; EMP: emphysema. ${ }^{\#}$ : mean value is different from mean of CON hamsters, $p \leqslant 0.1 ;{ }^{\uparrow}$ : mean value is different from mean of CON hamsters, $p \leqslant 0.05$. 
Table 2. - Muscle fibre cross-sectional area of type I, IIA, IIX, and IIB fibres

\begin{tabular}{|c|c|c|c|c|}
\hline Muscle & I & IIA & IIX & IIB \\
\hline \multicolumn{5}{|c|}{ Biceps femoris, caudal } \\
\hline $\mathrm{CON}$ & $1995 \pm 559$ & $2303 \pm 455$ & $3130 \pm 771$ & $5501 \pm 1363$ \\
\hline EMP & $1567 \pm 425$ & $1528 \pm 325^{\circ}$ & $2315 \pm 554^{\bullet}$ & $3889 \pm 538^{\circ}$ \\
\hline \multicolumn{5}{|c|}{ Vastus lateralis } \\
\hline $\mathrm{CON}$ & $2117 \pm 671$ & $1914 \pm 387$ & $3153 \pm 373$ & $3146 \pm 417$ \\
\hline EMP & $2165 \pm 565$ & $1812 \pm 346$ & $2312 \pm 360^{\circ}$ & $2434 \pm 564^{\circ}$ \\
\hline \multicolumn{5}{|c|}{ Gastrocnemius } \\
\hline $\mathrm{CON}$ & $1508 \pm 296$ & $1671 \pm 362$ & $2510 \pm 269$ & \\
\hline EMP & $1431 \pm 282$ & $1517 \pm 266$ & $1940 \pm 378^{\circ}$ & $1877 \pm 350$ \\
\hline \multicolumn{5}{|l|}{ Soleus } \\
\hline $\mathrm{CON}$ & $2045 \pm 959$ & $1629 \pm 710$ & & \\
\hline EMP & $1991 \pm 268$ & $1385 \pm 231$ & & \\
\hline \multicolumn{5}{|l|}{ Plantaris } \\
\hline $\mathrm{CON}$ & $1322 \pm 198$ & $2024 \pm 322$ & $2291 \pm 422$ & \\
\hline EMP & $1065 \pm 210^{\bullet}$ & $1381 \pm 248^{\bullet}$ & $1743 \pm 386^{\bullet}$ & \\
\hline \multicolumn{5}{|c|}{ Tibialis anterior } \\
\hline $\mathrm{CON}$ & $1136 \pm 97$ & $1291 \pm 421$ & $2789 \pm 757$ & $2978 \pm 376$ \\
\hline EMP & $1019 \pm 135$ & $886 \pm 33$ & $1498 \pm 215^{\#}$ & $1599 \pm 304^{\bullet}$ \\
\hline \multicolumn{5}{|c|}{ Diaphragm, costal } \\
\hline $\mathrm{CON}$ & $1150 \pm 210$ & $1166 \pm 119$ & $2269 \pm 325$ & \\
\hline EMP & $1343 \pm 359$ & $1517 \pm 409^{\#}$ & $2278 \pm 662$ & \\
\hline \multicolumn{5}{|c|}{ Diaphragm, crural } \\
\hline $\mathrm{CON}$ & $1498 \pm 235$ & $1314 \pm 189$ & $1786 \pm 451$ & \\
\hline EMP & $1480 \pm 432$ & $1570 \pm 333^{\#}$ & $2148 \pm 531$ & $1410 \pm 385$ \\
\hline
\end{tabular}

Data are presented as mean \pm SD given in $\mu \mathrm{m}^{2}$. CON: control; EMP: emphysema. ${ }^{\#}$ : mean value is different from mean of CON hamsters, $\mathrm{p} \leqslant 0.1 ;{ }^{\circ}$ : mean value is different from mean of CON hamsters, $\mathrm{p} \leqslant 0.05$.

correlation between lung pathology and skeletal muscle CSA. However, there was a trend for CSA of type IIA fibres to be increased in costal $(\mathrm{p}=0.06)$ and crural $(\mathrm{p}=0.1)$ portions of the diaphragm in EMP hamsters compared with CON.

EMP diminished the muscle weight of plantaris (EMP= $35.1 \pm 2.8 ; \quad \mathrm{CON}=39.5 \pm 5.7 \mathrm{mg} \quad(\mathrm{p}=0.03))$, tibialis anterior $(\mathrm{EMP}=91 \pm 14 ; \mathrm{CON}=139 \pm 2 \mathrm{mg}(\mathrm{p}=0.02))$, and costal diaphragm $(\mathrm{EMP}=242 \pm 27 ; \mathrm{CON}=289 \pm 27 \mathrm{mg} \quad(\mathrm{p}=0.002))$. All other muscle weights were similar between groups. Moreover, there was no significant correlation between muscle weights and CSA.

\section{Discussion}

The purpose of the present investigation was to determine whether EMP induces alterations in muscle fibre composition or induces atrophy in respiratory and locomotory muscles composed of different fibre types. The results demonstrate that EMP induced in hamsters stimulates only a modest transformation of locomotory muscle phenotype. The primary effect of this EMP model is atrophy of type I, IIA, IIX and IIB fibres in locomotory skeletal muscle. However, this effect is not uniform across all muscles. Fibre CSA in the tonically active diaphragm and soleus muscles was not altered by EMP. To the authors' knowledge, this is the first investigation to demonstrate fibre atrophy in locomotory skeletal muscles with diverse fibre composition and oxidative capacity in an animal model of EMP.

\section{Comparison with existing literature}

Fibre atrophy in EMP hamsters was induced in muscles with varying fibre makeup, ranging from 3-17\% type I fibres, 9-62\% type IIA fibres, $20-55 \%$ IIX fibres and $0-68 \%$ type IIB fibres (i.e. caudal biceps femoris, plantaris), as well as in muscles with diverse oxidative capacities, i.e. citrate synthase activity ranging from $20 \mu \mathrm{mol} \cdot \mathrm{min}^{-1} \cdot \mathrm{g}^{-1}$ in caudal biceps femoris to $52 \mu \mathrm{mol} \cdot \mathrm{min}^{-1} \cdot \mathrm{g}^{-1}$ in plantaris muscle [14] (table 2). In contrast, previous investigators have reported no differences in fibre CSA in plantaris muscle of EMP hamsters [15]. Because those investigators used a different methodology and fibre classification scheme (slow oxidative, fast oxidative glycolytic (FOG) and fast glycolytic), it could be argued that different methodologies may account for this disparity.

Conflicting reports of peripheral skeletal muscle atrophy in COPD patients also exist. HugHes et al. [10] reported reductions in type II, but not type I fibres in vastus lateralis muscle biopsies from COPD patients. In contrast, two other investigations demonstrated reductions in both type I and type II fibre size in peripheral skeletal muscle biopsies from COPD patients [7, 11]. Results from the present investigation demonstrating the variability of responses in muscle fibre CSA suggests that the contrasting results in COPD patients may be related to disparities in physical activity, pharmacological interventions or nutritional states among patient population studied.

\section{Potential mechanisms}

The underlying mechanism(s) inducing fibre atrophy within peripheral skeletal muscles remains uncertain. One possibility is that the fibre atrophy associated with EMP is secondary to physical inactivity and deconditioning. One line of evidence from the present investigation that lends support to this assertion is that those tonically active muscles used for respiration and the maintenance of posture, diaphragm and soleus muscles [16], respectively, did not experience fibre atrophy. All other limb muscles studied, which are recruited during locomotion $[13,16]$, demonstrated atrophy of one or more types of fibres in EMP hamsters. Thus, a decrement in physical activity would be expected to primarily affect these locomotory muscles and not tonically active muscles. Alternatively, tonically active muscles are by comparison 
predominately composed of type I fibres. Therefore, EMP may selectively induce fibre atrophy only in type II fibres in the absence of physical inactivity.

There are, however, several lines of evidence to indicate that inactivity alone cannot account for the peripheral skeletal muscle fibre atrophy that develops with EMP. First, previous work has demonstrated that activity levels are not different between CON hamsters and those with EMP [5]. Second, changes in muscle oxidative capacity are known to be associated with alterations in physical activity [17]. Previous work has demonstrated EMP reduces citrate synthase activity in hamster vastus lateralis and gastrocnemius muscles, but not the plantaris muscle [5]. If changes in citrate synthase activity and fibre atrophy are related to changes in activity of these muscles, then it would be expected that fibre atrophy would only occur in the vastus lateralis and gastrocnemius muscles and not the plantaris muscle. Moreover, type IIB fibres are only recruited during high-intensity exercise [13, 16], and it is these fibres that underwent some of the most profound atrophy (table 2). Therefore, if EMP-associated fibre atrophy were the result of reductions in cage activity, then fibre atrophy would be expected to primarily occur in type I, IIA and IIX fibres, but not type IIB fibres. However, this was not the case, as reductions in fibre CSA occurred in a muscle composed predominantly of type IIB fibres as well as type IIB fibres located within all other locomotory muscles.

Other possible mechanisms responsible for peripheral skeletal muscle alterations include hypoxia or malnutrition. Hypoxia has been demonstrated to induce slow-to-fast fibre transformation [18, 19], but what effect, if any, this has on fibre size remains unclear. ENGELEN et al. [20] have also implicated nutritional depletion in affecting the peripheral skeletal muscle function of COPD patients. Indeed, nutritional deprivation in animals causes certain skeletal muscle alterations, including decreased force production, increased fatigability, and type I and II fibre atrophy [21, 22]. It is possible that a reduced nutritional intake had an effect on EMP animals in the present investigation based upon their reduced body mass. Although, there were no weight differences in the majority of muscles analysed. Therefore, whether nutritional intake had an effect on animals in the present investigation is unknown. An additional mechanism underlying alterations in fibre size observed clinically includes pharmacological interventions. For example, myopathy is an established side-effect of corticosteroid treatment [23], which is consistent with the work of WILCOX et al. [24] demonstrating steroid treatment-induced type II fibre atrophy in peripheral skeletal muscle of EMP hamsters. However, corticosteroid treatment was not employed in the current investigation. Thus, it appears that a variety of factors may induce fibre atrophy in the peripheral skeletal muscles of COPD patients, but as demonstrated herein, physical inactivity, deconditioning and pharmacological intervention are not an obligatory part of this atrophic response.

The present investigation demonstrates that fibre composition is largely unaltered in the EMP hamster, the single exception being a decrease in the proportion of type IIB fibres in the tibialis anterior muscle (table 1), which may be explained by atrophy (table 2) and subsequent disappearance of these fibres. In the patient population, COPD has been demonstrated to induce variable alterations in fibre composition of peripheral skeletal muscles. Similar to the present investigation, Hughes et al. [10] and JAKOBSSON et al. [25] demonstrated no differences in fibre composition of vastus lateralis muscle biopsies from COPD patients, and SATO et al. [11] found no difference in fibre composition in biceps brachii muscle biopsies. In contrast, other investigators $[4,6,7]$ have reported that COPD patients have a lower proportion of type I fibres and an elevation in the per cent of type II fibres in the vastus lateralis muscle. In addition, two investigations [8, 9] have demonstrated altered $\mathrm{MyHC}$ expression in vastus lateralis muscle biopsies from COPD patients. These investigations collectively indicate that alterations in muscle fibre composition induced by COPD may differ between humans and animals.

\section{Respiratory muscles}

One consequence of EMP is that the diaphragm becomes mechanically disadvantaged and thus may have a greater energetic requirement [26] despite not performing more (or even as much) effective inspiratory work [27]. As indicated by LEVINE et al. [28], differences may exist between the adaptation of the diaphragm to EMP in animals and the human with COPD. In general, it appears that the hamster diaphragm adapts by increasing the CSA of both type I and II fibres without fibre transformation, whereas the human diaphragm adapts with type I fibre atrophy that may or may not be associated with a type II to I fibre transformation. However, FARKAS and Roussos [15] demonstrated atrophy of FOG fibres in diaphragm of EMP hamsters. This result may be related to the whole body weight loss demonstrated by their EMP hamsters. Data from the present investigation indicates there was a tendency for the diaphragm to adapt to EMP by increasing the CSA of type IIA fibres even though the weight of the costal portion decreased. Combined with reports of increased diaphragm capillarity [29, 30], these findings suggest that the diaphragm in EMP hamsters may undergo a compensatory response to improve its metabolic and mechanical efficiency.

In conclusion, the results of this investigation demonstrate that emphysema induces fibre atrophy in locomotory muscles and a tendency for hypertrophy in respiratory muscles with little or no change in muscle fibre composition. The atrophic response is not specific to locomotory muscles composed of a given fibre type or oxidative capacity, nor is it limited to a specific fibre population. Thus, therapeutic interventions should not be targeted at a given fibre type or muscle composed thereof, but inclusive of all. Although a variety of factors may induce fibre atrophy in the locomotory muscles of emphysema hamsters, physical inactivity or drug therapy per se do not appear to be an obligatory stimuli for this atrophic response.

\footnotetext{
Acknowledgements. The authors would like to gratefully thank the assistance of S. Miller for use of the imagining analysis system necessary for the completion of this project.
}

\section{References}

1. Jakobsson P, Jorfeldt L, Henriksson J. Metabolic enzyme activity in the quadriceps femoris muscle in patients with severe chronic obstructive pulmonary disease. Am J Respir Crit Care Med 1995; 151: 374-377.

2. Maltais F, Simard A, Simard C, Jobin J, Desgagnes P, LeBlanc P. Oxidative capacity of the skeletal muscle and lactic acid kinetics during exercise in normal subjects and in patients with COPD. Am J Respir Crit Care Med 1996; 153: 288-293.

3. Maltais F, Simard A, Simard C. Oxidative enzyme activities of the vastus lateralis muscle and the functional status in patients with COPD. Thorax 2000; 55: 848-853.

4. Gosker HR, van Mameren H, van Dijk PJ. Skeletal muscle fibre-type shifting and metabolic profile in patients with 
chronic obstructive pulmonary disease. Eur Respir J 2002; 19: 617-625.

5. Mattson JP, Poole DC. Pulmonary emphysema decreases hamster skeletal muscle oxidative enzyme capacity. $J$ Appl Physiol 1998; 85: 210-214.

6. Jobin J, Maltais F, Doyon JF. Chronic obstructive pulmonary disease: capillarity and fiber-type characteristics of skeletal muscle. J Cardiopulm Rehabil 1998; 18: 432-437.

7. Whittom F, Jobin J, Simard PM. Histochemical and morphological characteristics of the vastus lateralis muscle in patients with chronic obstructive pulmonary disease. Med Sci Sports Exerc 1998; 30: 1467-1474.

8. Satta A, Migliori GB, Spanevello A. Fibre types in skeletal muscles of chronic obstructive pulmonary disease patients related to respiratory function and exercise tolerance. Eur Respir J 1997; 10: 2853-2860.

9. Maltais F, Sullivan MJ, LeBlanc P. Altered expression of myosin heavy chain in the vastus lateralis muscle in patients with COPD. Eur Respir J 1999; 13: 850-854.

10. Hughes RL, Katz H, Sahgal V, Campbell JA, Hartz R, Shields TW. Fibre size and energy metabolites in five separate muscles from patients with chronic obstructive lung diseases. Respiration 1983; 44: 321-328.

11. Sato Y, Asoh T, Honda Y, Fujimatsu Y, Higuchi I, Oizumi K. Morphologic and histochemical evaluation of muscle in patients with chronic pulmonary emphysema manifesting generalized emaciation. Eur Neurol 1997; 37: 1116-1121.

12. Yoshikawa M, Yoneda $\mathrm{T}$, Takenaka H. Distribution of muscle mass and maximal exercise performance in patients with COPD. Chest 2001; 119: 93-98.

13. Delp MD, Duan C. Composition and size of type I, IIA, IID/X, and IIB fibers and citrate synthase activity of rat muscle. J Appl Physiol 1996; 80: 261-270.

14. Mattson JP, Miller TA, Poole DC, Delp MD. Fiber composition and oxidative capacity of hamster skeletal muscle. J Histochem Cytochem 2002; 50: 1685-1692.

15. Farkas GA, Roussos C. Histochemical and biochemical correlates of ventilatory muscle fatigue in emphysematous hamsters. J Clin Invest 1984; 74: 1214-1220.

16. Armstrong RB, Laughlin MH. Metabolic indicators of fibre recruitment in mammalian muscles during locomotion. $J$ Exp Biol 1985; 115: 201-213.

17. Saltin B, Gollnick PD. Skeletal muscle adaptability: significance for metabolism and performance. In: Peachey
LD, ed. Handbook of Physiology Section 10 Skeletal Muscle. Bethesda, American Physiological Society, 1983; pp. 555-631.

18. Itoh K, Moritani T, Ishida $\mathrm{K}$, Hirofuji $\mathrm{C}$, Taguchi S, Itoh M. Hypoxia-induced fibre type transformation in rat hindlimb muscles. Histochemical and electro-mechanical changes. Eur J Appl Physiol Occup Physiol 1990; 60: 331-336.

19. Hildebrand IL, Sylven C, Esbjornsson M, Hellstrom K, Jansson E. Does chronic hypoxaemia induce transformations of fibre types? Acta Physiol Scand 1991; 141: 435-439.

20. Engelen MP, Schols AM, Baken WC, Wesseling GJ, Wouters EF. Nutritional depletion in relation to respiratory and peripheral skeletal muscle function in out-patients with COPD. Eur Respir J 1994; 7: 1793-1797.

21. Russell DM, Atwood HL, Whittaker JS. The effect of fasting and hypocaloric diets on the functional and metabolic characteristics of rat gastrocnemius muscle. Clin Sci (Lond) 1984; 67: 185-194.

22. Sieck GC, Lewis MI, Blanco CE. Effects of undernutrition on diaphragm fiber size, SDH activity, and fatigue resistance. J Appl Physiol 1989; 66: 2196-2205.

23. Dekhuijzen PN, Decramer M. Steroid-induced myopathy and its significance to respiratory disease: a known disease rediscovered. Eur Respir J 1992; 5: 997-1003.

24. Wilcox PG, Hards JM, Bockhold K, Bressler B, Pardy RL. Pathologic changes and contractile properties of the diaphragm in corticosteroid myopathy in hamsters: comparison to peripheral muscle. Am J Respir Cell Mol Biol 1989; 1: 191199

25. Jakobsson $\mathrm{P}$, Jorfeldt L, Brundin A. Skeletal muscle metabolites and fibre types in patients with advanced chronic obstructive pulmonary disease (COPD), with and without chronic respiratory failure. Eur Respir J 1990; 3: 192-196.

26. Sexton WL, Poole DC. Effects of emphysema on diaphragm blood flow during exercise. J Appl Physiol 1998; 84: 971-979.

27. Sullivan KJ, Fournier M, Lewis MI. Respiratory work in elastase treated hamsters. Respir Physiol 1998; 114: 133-142.

28. Levine S, Gregory C, Nguyen T. Bioenergetic adaptation of individual human diaphragmatic myofibers to severe COPD. J Appl Physiol 2002; 92: 1205-1213.

29. Lewis MI, Zhan WZ, Sieck GC. Adaptations of the diaphragm in emphysema. J Appl Physiol 1992; 72: 934-943.

30. Poole DC, Mathieu-Costello O. Effect of pulmonary emphysema on diaphragm capillary geometry. J Appl Physiol 1997; 82: 599-606. 